“(C) 2015 IEEE. Personal use of this material is permitted. Permission from IEEE must be obtained for all other uses, in any current or future media, including reprinting/republishing this material for advertising or promotional purposes, creating new collective works, for resale or redistribution to servers or lists, or reuse of any copyrighted component of this work in other works." 


\title{
Integral Admittance Shaping for Exoskeleton Control
}

\author{
Umashankar Nagarajan $^{1}$, Gabriel Aguirre-Ollinger ${ }^{2}$ and Ambarish Goswami ${ }^{1}$
}

\begin{abstract}
A wide variety of strategies have been developed for assisting human locomotion using powered exoskeletons. Although these strategies differ in their aims as well as the control methods employed, they have the implicit property of causing a virtual modification of the dynamic response of the human limb. We use this property of the exoskeletons action to formulate a unified control design framework called Integral Admittance Shaping, which designs exoskeleton controllers capable of producing the desired dynamic response for the assisted limb. In this framework, a virtual increase in the admittance of the limb is produced by coupling it to an exoskeleton that exhibits active behavior. Specifically, our framework shapes the magnitude profile of the integral admittance (i.e. torque-to-angle relationship) of the coupled human-exoskeleton system, such that the desired assistance is achieved. This framework also ensures that the coupled stability and passivity are guaranteed. This paper presents a formulation of Integral Admittance Shaping for single degree-of-freedom (1-DOF) exoskeleton devices. We also present experimental results on a modified version of Honda's Stride Management Assist (SMA) device that successfully demonstrate motion amplification of the assisted hip joint during walking.
\end{abstract}

\section{INTRODUCTION}

Exoskeletons are electromechanical devices that physically and energetically interact with human limbs to provide assistance to their motions [1], [2]. The exoskeleton literature contains a wide variety of strategies that assist human locomotion. The strategies that focus on dynamics and energetics of human walking attempt to achieve one of the following three objectives:

1) Metabolic cost reduction: Tethered exoskeleton devices with off-board power and actuation have been used to demonstrate reduction in metabolic cost for human walking [3], [4]. The first autonomously powered exoskeleton device that experimentally demonstrated reduction in metabolic cost for human walking during load carriage was presented recently in [5]. However, to the best of our knowledge, no autonomously powered exoskeleton device has so far experimentally demonstrated a reduction in metabolic cost for walking or running.

2) Muscle effort reduction: Feedback controllers based on electromyographic (EMG) signals were used to provide torque assist to the hip, knee and ankle joints during locomotion in [6]. It was experimentally demonstrated in [7] that while walking at a given speed, humans automatically learn

*This work was supported by Honda Research Institute USA Inc.

${ }^{1}$ U. Nagarajan and A. Goswami are with Honda Research Institute USA, Inc., Mountain View, CA, 94043 USA unagarajan@hra.com, agoswami@hra.com

${ }^{2}$ G. Aguirre-Ollinger is with the School of Electrical, Mechanical and Mechatronic Systems, University of Technology, Sydney, Broadway, NSW 2007 Australia gabriel.aguirre-ollingereuts.edu.au to reduce their muscle activations when appropriate assistive torques are added to their ankle joints. Several exoskeletons have also been designed to achieve increased load-carrying capabilities [8], [9].

3) Walking speed increase: Powered ankle-foot orthoses were used in [10] to demonstrate that the preferred walking speed of humans can be potentially increased by providing assistive torques at the ankle joints. Ankle exoskeletons have been used to demonstrate increase in step lengths while keeping the step frequency constant [11]. EMG-based control was used to modify the natural frequency of the leg around the knee joint in [12]. Active impedance control was used to experimentally demonstrate increase in natural frequency of free-leg swinging at the knee joint [13].

Although the assistive strategies listed above are different in their aims and control methods involved, they have one thing in common. They all cause a virtual modification of the dynamic response of the human limb. Each strategy modifies the coupled human-exoskeleton system dynamics to achieve a desired dynamic response that produces a particular benefit like reduced muscle effort or increased walking speed. Exploiting this common feature, we present Integral Admittance Shaping, a unified control design framework that designs exoskeleton controllers capable of producing the desired dynamic response for the assisted limb.

Needless to say, the exoskeleton does not change the physical properties of the human limb as such. The modification of the limb's dynamic response is a virtual effect caused by the exoskeleton's active behavior. The specific goal of our framework is to shape the integral admittance (torque-toangle relationship) of the coupled system. Thus, we define assistance as an overall increase in the magnitude of the integral admittance, and resistance as an overall decrease thereof. Our framework can be used to achieve any desired frequency response magnitude profile that satisfies the user's needs. A critical feature is that it ensures that the coupled human-exoskeleton system is both stable and passive in spite of the exoskeleton's active behavior.

In this work, we focus on single-joint motion and use linearized models of both the exoskeleton and the human limb. Even though we focus on lower-extremity exoskeletons in this work, our control design framework can be used for upper-extremity exoskeletons as well. We also present successful experimental results on a modified version of Honda's Stride Management Assist (SMA) device, a hip exoskeleton device with two 1-DOF joints as shown in Fig. 1. 


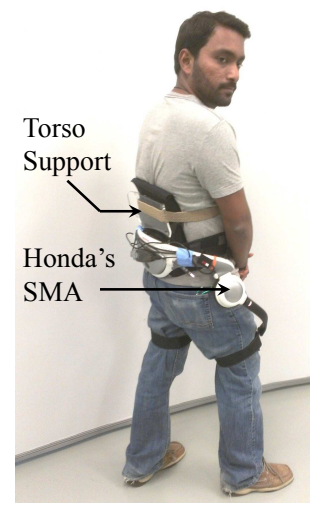

Fig. 1. A human subject with a modified version of Honda's Stride Management Assist (SMA) exoskeleton device [14] with two 1-DOF hip joints. A torso support was added to the SMA device to provide greater damping and reduce oscillations when the motor torques were large.

\section{LineAR JoINT DyNAMiCS OF A COUPLED HuMAN-EXosKeLETON SYSTEM WITH SOFT COUPLING}

In this work, we use an elementary model that consists of linearized 1-DOF models for the human leg and the exoskeleton. The 1-DOF leg model is an approximation of the extended leg with locked-knee swinging about the hip joint. The coupling between the exoskeleton and the human limb can be either rigid (Fig. 2(a)) or soft (Fig. 2(b)). The human limb and the exoskeleton cannot move relative each other with rigid coupling, whereas with soft coupling, they can. Any realistic and practical exoskeleton device will be attached to the limb, wherein the intervening muscle, tissue, fat and other body substances produce a soft coupling between the exoskeleton device and the bone as shown in Fig. 2(b). This soft coupling can be modeled with a linear torsional spring with coefficient $k_{c}$ and a linear torsional damper with coefficient $b_{c}$ as shown in Fig. 3.

The linear equations of motion of an isolated 1-DOF human joint are given by

$$
I_{h} \ddot{\theta}_{h}(t)+b_{h} \dot{\theta}_{h}(t)+k_{h} \theta_{h}(t)=\tau_{h}(t),
$$

where $\theta_{h}(t)$ is the joint angle trajectory, $I_{h}, b_{h}, k_{h}$ are the associated moment of inertia, joint damping coefficient and joint stiffness coefficient respectively, and $\tau_{h}(t)$ is the joint torque trajectory. The stiffness term $k_{h} \theta_{h}(t)$ will include the linearized gravitational terms. Similarly, the linear equations of motion of an isolated 1-DOF exoskeleton are given by

$$
I_{e} \ddot{\theta}_{e}(t)+b_{e} \dot{\theta}_{e}(t)+k_{e} \theta_{e}(t)=\tau_{e}(t),
$$

where $\theta_{e}(t)$ is the joint angle trajectory, $I_{e}, b_{e}, k_{e}$ are the associated moment of inertia, joint damping coefficient and joint stiffness coefficient respectively, and $\tau_{e}(t)$ is the joint torque trajectory.

The linear equations of motion of a coupled humanexoskeleton system with soft coupling are given by

$$
\begin{aligned}
I_{h} \ddot{\theta}_{h}(t)+b_{h} \dot{\theta}_{h}(t)+k_{h} \theta_{h}(t) & =\tau_{h}(t)-\tau_{c}(t), \\
I_{e} \ddot{\theta}_{e}(t)+b_{e} \dot{\theta}_{e}(t)+k_{e} \theta_{e}(t) & =\tau_{e}(t)+\tau_{c}(t),
\end{aligned}
$$

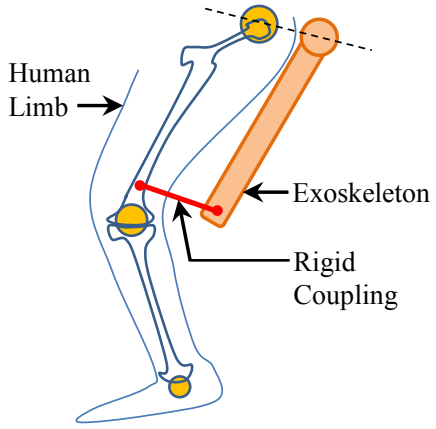

(a)

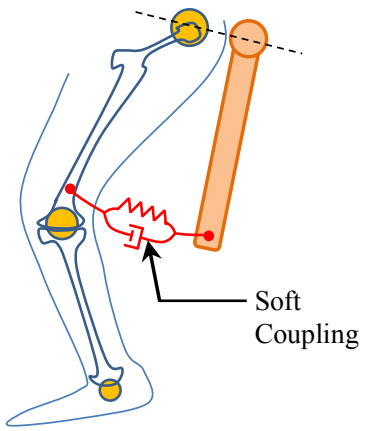

(b)
Fig. 2. An elementary model with: (a) rigid coupling, and (b) soft coupling.

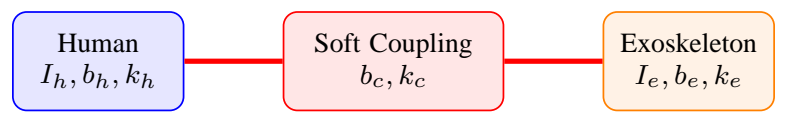

Fig. 3. Coupled human-exoskeleton system with soft coupling. Secondorder linear models are used to represent the joint dynamics of the human (Eq. 1) and the exoskeleton (Eq. 2).

where $\tau_{c}$ is the coupling torque at the joint corresponding to the interaction force between exoskeleton and the human limb, and it is given by

$$
\tau_{c}(t)=b_{c}\left(\dot{\theta}_{h}(t)-\dot{\theta}_{e}(t)\right)+k_{c}\left(\theta_{h}(t)-\theta_{e}(t)\right) .
$$

In this work, we aim at modifying the joint dynamics of the coupled human-exoskeleton system by modifying the impedance and admittance of the coupled system. The following section reviews the definitions of impedance, admittance and introduces integral admittance, which will be used extensively in the rest of the paper.

\section{A. Impedance, Admittance and Integral Admittance}

For the linear human joint dynamics in (1), its impedance transfer function $Z_{h}(s)$ [15] is given by

$$
Z_{h}(s)=\frac{\tau_{h}(s)}{\Omega_{h}(s)}=\frac{I_{h} s^{2}+b_{h} s+k_{h}}{s},
$$

and its admittance transfer function $Y_{h}(s)$ [15] is given by

$$
Y_{h}(s)=\frac{\Omega_{h}(s)}{\tau_{h}(s)}=\frac{s}{I_{h} s^{2}+b_{h} s+k_{h}},
$$

where $\Omega_{h}(s)$ is the Laplace transform of $\dot{\theta}_{h}(t)$, and $\tau_{h}(s)$ is the Laplace transform of $\tau_{h}(t)$. For a linear system, its impedance is the inverse of its admittance and vice-versa.

We define integral admittance transfer function $X_{h}(s)$ as the integral of the admittance transfer function, and it is given by

$$
X_{h}(s)=\frac{\Theta_{h}(s)}{\tau_{h}(s)}=\frac{1}{I_{h} s^{2}+b_{h} s+k_{h}},
$$

where $\Theta_{h}(s)$ is the Laplace transform of $\theta_{h}(t)$.

The admittance transfer function of an isolated exoskeleton $Y_{e}(s)$ is given by

$$
Y_{e}(s)=\frac{\Omega_{e}(s)}{\tau_{e}(s)}=\frac{s}{I_{e} s^{2}+b_{e} s+k_{e}},
$$




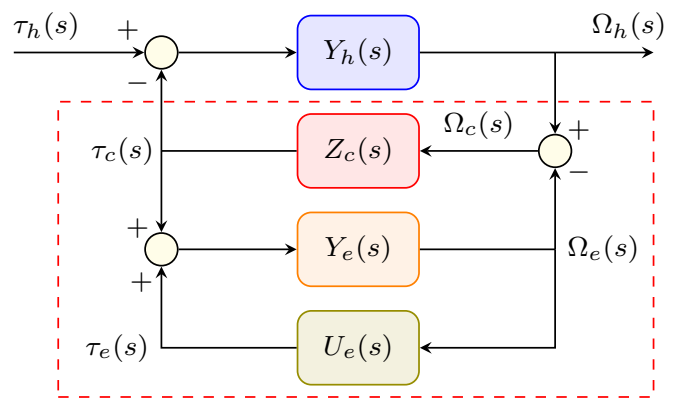

(a)

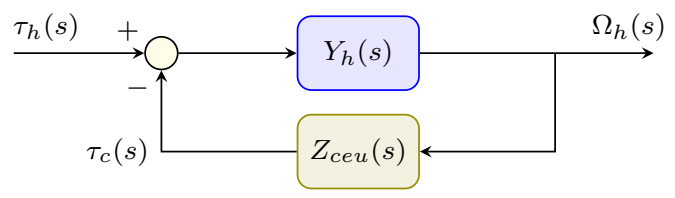

(b)

Fig. 4. Block diagram of the coupled human-exoskeleton system with the exoskeleton controller $U_{e}(s)$.

and the impedance transfer function of an isolated coupling element $Z_{c}(s)$ is given by

$$
Z_{c}(s)=\frac{\tau_{c}(s)}{\Omega_{c}(s)}=\frac{b_{c} s+k_{c}}{s},
$$

where $\Omega_{c}(s)=\Omega_{h}(s)-\Omega_{e}(s)$.

\section{B. Closed-loop Coupled Human-Exoskeleton System}

For any exoskeleton controller $U_{e}(s)$ that feeds back the exoskeleton joint angular velocity $\Omega_{e}(s)$, the closed-loop coupled human-exoskeleton system can be represented as a block diagram in Fig. 4(a).

The outlined region in Fig. 4( $a)$ containing $Z_{c}(s), Y_{e}(s)$ and $U_{e}(s)$ can be reduced to a single transfer function $Z_{\text {ceu }}(s)$ given by

$$
Z_{c e u}(s)=\frac{Z_{c}(s)}{1+Z_{c}(s) Y_{e u}(s)},
$$

as shown in Fig. 4(b). Here, the transfer function $Y_{e u}(s)$ is given by

$$
Y_{e u}(s)=\frac{Y_{e}(s)}{1-Y_{e}(s) U_{e}(s)} .
$$

The loop transfer function $L_{h e u}(s)$ needed to evaluate the stability of the feedback system shown in Fig. 4(b) is given by

$$
L_{h e u}(s)=Y_{h}(s) Z_{c e u}(s) \text {, }
$$

and the gain margin $G M$ of $L_{h e u}(s)$ is given by

$$
G M\left(L_{h e u}\right)=\frac{1}{\left|L_{h e u}\left(j \omega_{c}\right)\right|},
$$

where $\omega_{c}$ is the phase-crossover frequency when the phase of $L_{h e u}(s)$ is $180^{\circ}$, i.e., $\angle L_{h e u}\left(j \omega_{c}\right)=180^{\circ}$. The gain margin $G M\left(L_{h e u}\right)$ is the maximum positive gain exceeding which the closed-loop system becomes unstable. Therefore, in order for the coupled human-exoskeleton system shown in Fig. 4(b) to be stable, the following condition needs to be satisfied:

$$
G M\left(L_{\text {heu }}\right)>1 .
$$

From Fig. 4(b), the closed-loop admittance $Y_{\text {heu }}(s)$ of the coupled human-exoskeleton system with the exoskeleton controller $U_{e}(s)$ is given by

$$
Y_{h e u}(s)=\frac{Y_{h}(s)}{1+Y_{h}(s) Z_{c e u}(s)},
$$

and its corresponding closed-loop integral admittance $X_{h e u}(s)$ is given by

$$
X_{\text {heu }}(s)=\frac{Y_{\text {heu }}(s)}{s}=\frac{X_{h}(s)}{1+Y_{h}(s) Z_{\text {ceu }}(s)},
$$

where $X_{h}(s)=Y_{h}(s) / s$ as shown in (8).

In addition to coupled stability, another important requirement for dynamically interacting systems is coupled passivity [16], which ensures that the coupled human-exoskeleton system does not become unstable when in contact with any passive environment [17], for example when the foot contacts the ground. From [16], it can be shown that in order for a stable coupled human-exoskeleton system satisfying (15) to be passive, the following phase condition needs to be satisfied:

$$
\angle X_{h e u}(j \omega) \in\left[-180^{\circ}, 0^{\circ}\right] \quad \forall \omega .
$$

\section{INTEGRAL ADMITTANCE SHAPING}

In this section, we present Integral Admittance Shaping, a unified control design framework that designs exoskeleton controllers which shape the frequency response magnitude of the closed-loop integral admittance $X_{h e u}(s)$ of the coupled human-exoskeleton system in (17) such that the 1-DOF human joint is assisted. In order to design the shape of $\left|X_{h e u}(j \omega)\right|$, one needs to define the desired objective of the exoskeleton. This work focuses on providing assistance and avoiding resistance, and hence we first define assistance and resistance in a clear and quantitative way.

\section{A. Assistance and Resistance}

This section begins with conceptual definitions of assistance and resistance, and proceeds to present their quantitative definitions.

Definition 1: A 1-DOF human joint is said to be assisted (resisted) by an exoskeleton if the frequency response magnitude of the integral admittance of the coupled humanexoskeleton system is greater (lesser) than that of the unassisted human for all frequencies of interest.

According to the above definition, a human joint is assisted when $\left|X_{h e u}(j \omega)\right|>\left|X_{h}(j \omega)\right|$, and it is resisted when $\left|X_{h e u}(j \omega)\right|<\left|X_{h}(j \omega)\right|$. An assistive exoskeleton behavior produces motion amplification, i.e., for a given input joint torque, the amplitude of the joint angular motion is larger than that without assist, and torque reduction, i.e., the amplitude of joint torque required to achieve a particular joint angle motion is smaller than that required without assist. 


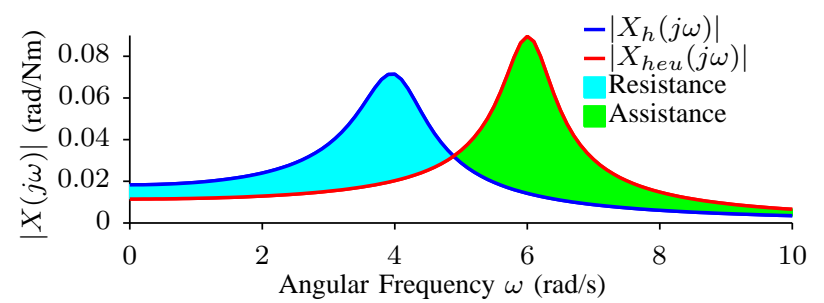

(a)

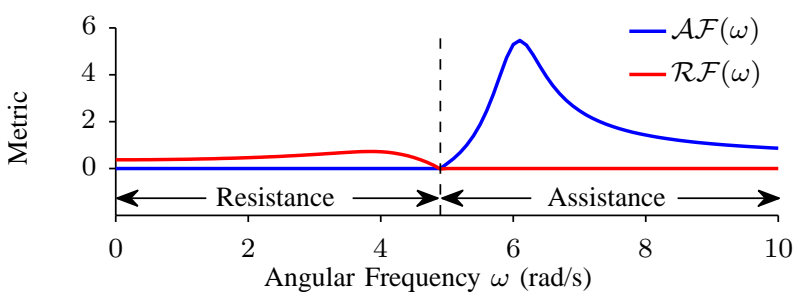

(b)

Fig. 5. Assistance and Resistance of a 1-DOF assistive exoskeleton represented in terms of: (a) Integral Admittance $X(s)$, and $(b)$ Assistance Function $\mathcal{A F}(\omega)$ and Resistance Function $\mathcal{R} \mathcal{F}(\omega)$.

Conversely, motion reduction and torque amplification are the characteristics of a resistive exoskeleton behavior.

Figure 5(a) shows the frequency response magnitude plots of an unassisted human and a hypothetical coupled humanexoskeleton system whose parameters are listed in Table I (Appendix I). As per Definition 1, the green colored regions represent the frequencies where the coupled system experiences assistance, i.e., $\left|X_{h e u}(j \omega)\right|>\left|X_{h}(j \omega)\right|$, and the cyan colored regions represent the frequencies where resistance, i.e., $\left|X_{h e u}(j \omega)\right|<\left|X_{h}(j \omega)\right|$, is experienced.

Figure 5 shows that at each frequency $\omega$, the exoskeleton behavior could be either only assistive or only resistive, but cannot be both. Based on this observation, the assistance function $\mathcal{A} \mathcal{F}(\omega)$ is defined as

$\mathcal{A F}(\omega)=\left\{\begin{array}{cl}\frac{\left|X_{h e u}(j \omega)\right|-\left|X_{h}(j \omega)\right|}{\left|X_{h}(j \omega)\right|} & \text { if }\left|X_{h e u}(j \omega)\right| \geq\left|X_{h}(j \omega)\right| \\ 0 & \text { if }\left|X_{h e u}(j \omega)\right|<\left|X_{h}(j \omega)\right|,\end{array}\right.$

and the resistance function $\mathcal{R} \mathcal{F}(\omega)$ is defined as

$\mathcal{R} \mathcal{F}(\omega)=\left\{\begin{array}{cl}0 & \text { if }\left|X_{h e u}(j \omega)\right| \geq\left|X_{h}(j \omega)\right| \\ \frac{\left|X_{h}(j \omega)\right|-\left|X_{h e u}(j \omega)\right|}{\left|X_{h}(j \omega)\right|} & \text { if }\left|X_{h e u}(j \omega)\right|<\left|X_{h}(j \omega)\right| .\end{array}\right.$

At any frequency $\omega$, the assistance function $\mathcal{A} \mathcal{F}(\omega) \in[0, \infty]$, and the resistance function $\mathcal{R} \mathcal{F}(\omega) \in[0,1]$. When the coupled human-exoskeleton joint dynamics is identical to the unassisted natural human joint dynamics, i.e., $\left|X_{\text {heu }}(j \omega)\right|=$ $\left|X_{h}(j \omega)\right|$, then $\mathcal{A} \mathcal{F}(\omega)=\mathcal{R} \mathcal{F}(\omega)=0, \forall \omega$. The upper bound $\mathcal{A F}(\omega)=\infty$ is achieved when $\left|X_{\text {heu }}(j \omega)\right|=\infty$, and the upper bound $\mathcal{R} \mathcal{F}(\omega)=1$ is achieved when $\left|X_{\text {heu }}(j \omega)\right|=0$.

Using the assistance function $\mathcal{A F}(\omega)$ and the resistance function $\mathcal{R} \mathcal{F}(\omega)$, this section defines the following quantitative metrics for assistance and resistance of a 1-DOF assistive exoskeleton, namely assistance ratio and resistance ratio.
Definition 2: Assistance Ratio $\mathcal{A}$ is defined as the average value of the assistance function $\mathcal{A F}(\omega)$ over a range of frequencies $\left[0, \omega_{f}\right]$, and is given by

$$
\mathcal{A}=\frac{1}{\omega_{f}} \int_{0}^{\omega_{f}} \mathcal{A} \mathcal{F}(\omega) d \omega
$$

Definition 3: Resistance Ratio $\mathcal{R}$ is defined as the average value of the resistance function $\mathcal{R} \mathcal{F}(\omega)$ over a range of frequencies $\left[0, \omega_{f}\right]$, and is given by

$$
\mathcal{R}=\frac{1}{\omega_{f}} \int_{0}^{\omega_{f}} \mathcal{R} \mathcal{F}(\omega) d \omega
$$

Similar to the assistance and resistance functions, the assistance ratio $\mathcal{A} \in[0, \infty]$ and the resistance ratio $\mathcal{R} \in$ $[0,1]$. As described earlier, the upper bounds $\mathcal{A}=\infty$ and $\mathcal{R}=1$ can be achieved only if $\left|X_{h e u}(j \omega)\right|=\infty$ and $\left|X_{a}(j \omega)\right|=0$ respectively $\forall \omega$. Although these bounds are mathematically valid, they are not realistic for any proper integral admittance transfer function.

With the above definitions of assistance and resistance, the following section enumerates the desired characteristics of an assisted coupled human-exoskeleton system.

\section{B. Desired Characteristics of an Assisted Coupled Human- Exoskeleton System}

The primary objective of an assistive exoskeleton is to provide assistance to any human motion and not to resist any motion. However, it is vital to ensure that the coupled humanexoskeleton system is also stable and passive. Therefore, the desired characteristics of a 1-DOF assisted coupled humanexoskeleton system can be listed as follows:

1) Coupled Stability, i.e., $G M\left(L_{h e u}\right)>1$;

2) Coupled Passivity, i.e., $/ X_{h e u}(j \omega) \in\left[-180^{\circ}, 0^{\circ}\right], \forall \omega$;

3) Positive Assistance, i.e. $\mathcal{A}>0$; and

4) No Resistance, i.e. $\mathcal{R}=0$.

The above characteristics are only the necessary characteristics, and more desired characteristics can be added depending on the task and its goal. It is important to note that although the coupled human-exoskeleton system is desired to be passive, the isolated exoskeleton system with its controller need not be passive. In fact, the exoskeleton has to exhibit active behavior in order to provide any positive assistance as will be shown in the next section.

\section{Exoskeleton Control Law}

The preceeding sections provided the necessary metrics to evaluate assistance and also enumerated the desired characteristics of an assisted coupled human-exoskeleton system. Now, one can proceed to design an exoskeleton controller $U_{e}(s)$ that actually shapes the closed-loop integral admittance of the coupled system based on these metrics. We first present an intuitive approach to designing such a controller.

If the desired exoskeleton dynamics is given by a desired moment of inertia $I_{e}^{d}$, a desired joint damping coefficient $b_{e}^{d}$ 
and a desired joint stiffness coefficient $k_{e}^{d}$, then the exoskeleton torque $\tau_{e}$ required to achieve the desired exoskeleton dynamics can be derived from (2) as follows:

$\tau_{e}(t)=\left(I_{e}-I_{e}^{d}\right) \ddot{\theta}_{e}(t)+\left(b_{e}-b_{e}^{d}\right) \dot{\theta}_{e}(t)+\left(k_{e}-k_{e}^{d}\right) \theta_{e}(t)$.

It can be verified that the control law in (23) reduces the isolated exoskeleton dynamics in (2) to

$$
I_{e}^{d} \ddot{\theta}_{e}(t)+b_{e}^{d} \dot{\theta}_{e}(t)+k_{e}^{d} \theta_{e}(t)=0,
$$

as desired. The exoskeleton controller $U_{e}^{0}(s)$ corresponding to the control law in (23) that feeds back angular velocity $\Omega_{e}(s)$ is given by

$$
U_{e}^{0}(s)=\frac{K_{\alpha} s^{2}+K_{\omega} s+K_{\theta}}{s},
$$

where $K_{\alpha}=I_{e}-I_{e}^{d}, K_{\omega}=b_{e}-b_{e}^{d}$, and $K_{\theta}=k_{e}-k_{e}^{d}$ are the feedback gains on angular acceleration $\ddot{\theta}_{e}$, angular velocity $\dot{\theta}_{e}$ and angle $\theta_{e}$ respectively. Equation (25) is the first intuitive formulation of the exoskeleton controller, and we now discuss its properties and limitations.

This work defines an exoskeleton to be assistive if it increases the admittance of the coupled human-exoskeleton system above that of the unassisted human limb. In other words, the impedance of the coupled human-exoskeleton system needs to be reduced below that of the unassisted human limb. This implies that the exoskeleton needs to cancel its own impedance first and then compensate for at least a part of the human limb's impedance. Therefore, the desired exoskeleton behavior must be that of a negative impedance, i.e., $I_{e}^{d}<0, b_{e}^{d}<0$ and $k_{e}^{d}<0$. Consequently, the feedback gains $K_{\alpha}, K_{\omega}$ and $K_{\theta}$ in (25) will all be positive. In other words, the exoskeleton controller uses positive feedback, and hence the exoskeleton exhibits active behavior, which is capable of performing net positive work on the limb.

However, positive feedback naturally raises the question of stability, and so we now explain how coupled stability can be achieved. Although the exoskeleton exhibits active behavior, which can be potentially destabilizing, the controller can be designed such that the coupled human-exoskeleton system is stable and passive. Our earlier work in [18], [19] show that stiffness compensation and damping compensation can be successfully achieved with positive feedback while guaranteeing stability of the coupled system. However, inertia compensation is more complex and ultimately requires a modification of the controller in (25).

It can be shown that using only positive acceleration feedback as in (25), the gain margin of the coupled system reduces to the moment of inertia of the exoskeleton, which implies that the exoskeleton controller in (25) can at the most compensate for the exoskeleton's own moment of inertia before going unstable. This implies that the moment of inertia of the coupled human-exoskeleton system cannot be reduced below that of the unassisted human limb, without compromising coupled stability. However, using low-pass

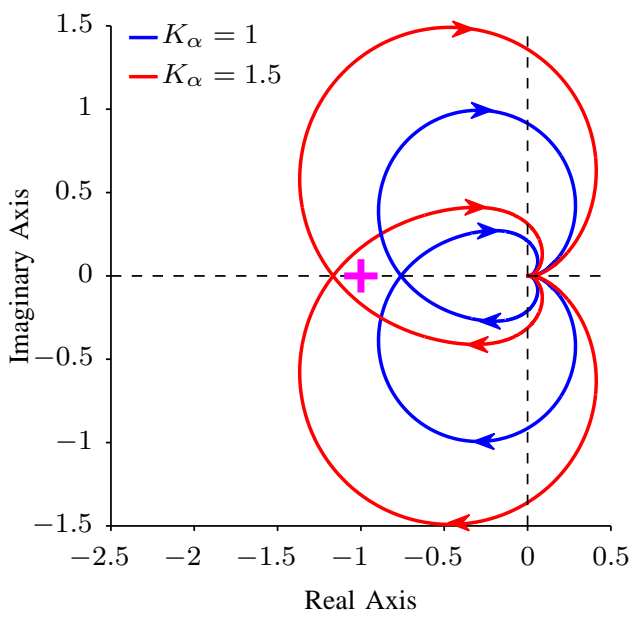

Fig. 6. Nyquist plots of $L_{h e u}(s)$ for $K_{\alpha}=1$ and $K_{\alpha}=1.5$, while the other parameters were fixed at $K_{\theta}=K_{\omega}=0$ and $\omega_{l o}=10 \mathrm{rad} / \mathrm{s}$.

filtered acceleration feedback, it can be shown that inertia reduction can be achieved. The proofs for the above statements are beyond the scope of this paper and will be detailed in a future publication [20]. Thus, this work uses filtered acceleration feedback with a second-order low-pass Butterworth filter $H_{l o}(s)$ with a cut-off frequency $\omega_{l o}$ given by

$$
H_{l o}(s)=\frac{\omega_{l o}^{2}}{s^{2}+\sqrt{2} \omega_{l o} s+\omega_{l o}^{2}},
$$

which results in a modified control transfer function $U_{e}(s)$ given by

$$
U_{e}(s)=\frac{K_{\alpha} H_{l o}(s) s^{2}+K_{\omega} s+K_{\theta}}{s} .
$$

Figure 6 shows the Nyquist plots of $L_{h e u}(s)$ with the exoskeleton controller $U_{e}(s)$ in (27) for $K_{\alpha}=1$ and $K_{\alpha}=$ 1.5 , while the remaining control parameters were fixed at $K_{\theta}=K_{\omega}=0$ and $\omega_{l o}=10 \mathrm{rad} / \mathrm{s}$. Since $L_{\text {heu }}(\mathrm{s})$ has no unstable poles, the number of encirclements of the Nyquist plot around $-1+j 0$ is sufficient to determine the stability of the closed-loop system. For $K_{\alpha}=1$, its Nyquist plot does not encircle $-1+j 0$ resulting in a stable closed-loop system, whereas for $K_{\alpha}=1.5$, its Nyquist plot encircles $-1+j 0$ twice resulting in an unstable closed-loop system.

Figure 6 emphasizes that there exists a set of control parameters $\left\{K_{\theta}, K_{\omega}, K_{\alpha}, \omega_{l o}\right\}$ for which the exoskeleton emulates a negative impedance and exhibits active behavior while still guaranteeing the stability of the coupled human-exoskeleton system. The control parameters $\left\{K_{\theta}, K_{\omega}, K_{\alpha}, \omega_{l o}\right\}$ directly affect the closed-loop integral admittance $X_{\text {heu }}(s)$, and they can be chosen such that the frequency response magnitude profile of $X_{\text {heu }}(s)$ is shaped such that the desired assistance $\mathcal{A}_{d}$ as defined in Sec. III-A is achieved. The following section presents an optimization algorithm that finds a set of control parameters to achieve the desired assistance $\mathcal{A}_{d}$. 


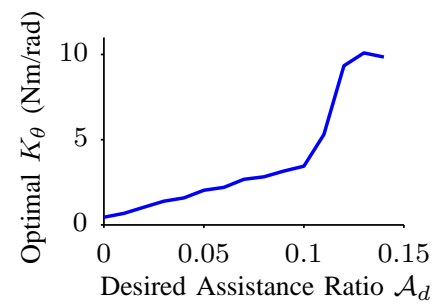

(a)

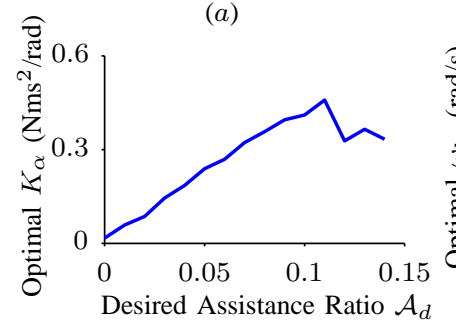

(c)

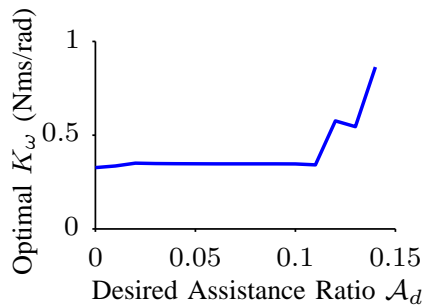

(b)

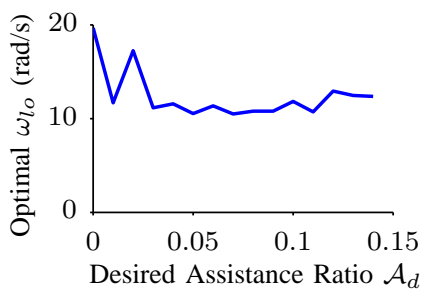

(d)
Fig. 7. Optimal control parameters needed to achieve $\mathcal{A}_{d} \in[0,0.1422]$ : (a) $K_{\theta},(b) K_{\omega},(c) K_{\alpha}$ and (d) $\omega_{l o}$.

\section{Optimization to Achieve a Desired Assistance}

Given a desired assistance ratio $\mathcal{A}_{d}$, the optimal set of control parameters of the 1-DOF coupled human-exoskeleton system in (17) can be obtained using the following constrained optimization problem:

$$
\begin{aligned}
& \underset{\left\{K_{\theta}, K_{\omega}, K_{\alpha}, \omega_{l o}\right\}}{\operatorname{minimize}}\left|\mathcal{A}-\mathcal{A}_{d}\right|^{2}+w \mathcal{R} \\
& \text { subject to } \quad G M\left(L_{\text {heu }}\right)>1 \text {, } \\
& \begin{array}{l}
\frac{X_{h e u}(j \omega)}{\left|\frac{\zeta_{\text {heu }}-\zeta_{h}}{\zeta_{h}}\right|} \in\left[-180^{\circ}, 0^{\circ}\right] \quad \forall \omega, \\
<\epsilon .
\end{array}
\end{aligned}
$$

Here, $\zeta_{h e u}$ and $\zeta_{h}$ are the damping ratios of the dominant second-order dynamics of the closed-loop system and the isolated human limb respectively, and the maximum permissible ratio of change in the damping ratio is given by $\epsilon$.

Instead of using a hard constraint of $\mathcal{R}=0$, the optimization in (28) uses a soft constraint error term weighted by $w$ in the objective function so that the optimizer has more freedom to explore the parameter space. Using a large weight $w$, the optimizer will eventually converge to control parameters that result in either zero resistance ratio $(\mathcal{R}=0)$ or negligible resistance ratio $(\mathcal{R} \approx 0)$. In addition to the coupled stability and passivity constraints, the optimization in (28) also has a constraint on the damping ratio. Since the oscillatory behavior of the dominant second-order dynamics of the coupled human-exoskeleton system is determined by its damping ratio $\zeta_{h e u}$, and its variation is restricted in order to provide greater comfort to the user.

In this work, the optimizations were performed in Matlab using fminsearch() function, which in turn used Nelder-Mead simplex algorithm [21]. Nelder-Mead simplex algorithm is a heuristic search method that uses only function evaluations to solve unconstrained optimization problems. Constraints were enforced indirectly by using large cost function values

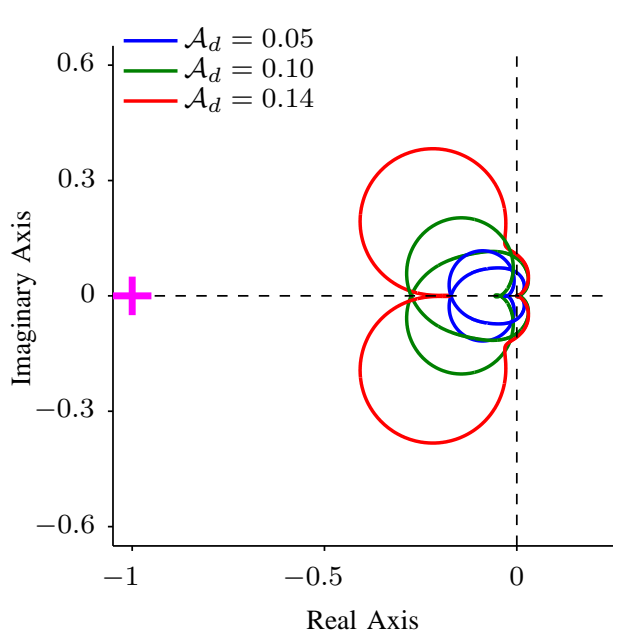

Fig. 8. Nyquist plots of $L_{h e u}(s)$ for the optimal control parameters in Fig. 7 that achieve coupled stability and passivity.

$\left(10^{5}\right)$ when the constraints were not satisfied, and the soft constraint weight was chosen to be $w=10^{5}$. The functional and parameter tolerances were both set to $10^{-5}$.

Figure 7 shows the derived optimal control parameters for the different desired assistance ratios $\mathcal{A}_{d} \leq 0.1422$ that satisfy both the coupled stability and passivity constraints. For $\mathcal{A}_{d}>0.1422$, the optimization was unable to find control parameters that guaranteed both coupled stability and passivity. Here, the optimal control parameters for $\mathcal{A}_{d}=0$ $\left(\left|X_{\text {heu }}(j \omega)\right|=\left|X_{h}(j \omega)\right|\right)$ were first obtained, and then the optimal control parameters for $\mathcal{A}_{d}$ were chosen as the initial optimization parameters for $\mathcal{A}_{d}^{\prime}=\mathcal{A}_{d}+0.01$. Therefore, the optimization converges to the control parameters closest to those that match the integral admittance magnitude profile of the unassisted human.

Figure 8 shows the Nyquist plots of $L_{h e u}(s)$ corresponding to a few selected optimal control parameters shown in Fig. 7. None of the Nyquist plots encircle $-1+j 0$, thereby implying that the derived coupled human-exoskeleton systems are all stable. Their corresponding integral admittance magnitude and phase plots are shown in Fig. 9. Figure 9(b) shows that the coupled systems satisfy the passivity condition $\angle X_{h e u}(j \omega) \in\left[-180^{\circ}, 0^{\circ}\right]$, given in (18).

\section{EXPERIMENTAL RESULTS}

This section presents experimental results that evaluate the integral admittance shaping framework on a modified version of the Honda's Stride Management Assist (SMA) device, which was shown in Fig. 1. The SMA device is a hip exoskeleton with two 1-DOF joints, one for each leg. A torso support was added to the device in order to provide greater damping and reduce oscillations were the motor torques were large. Appendix I presents the system parameters of the exoskeleton device in Table I. At each joint, the SMA device has a hall effect sensor that measures the exoskeleton joint angle $\theta_{e}$, and a DC motor with a torque saturation of $6 \mathrm{Nm}$.

Figure 10 presents the control framework that executes the exoskeleton control in Sec. III-C using the optimized control 


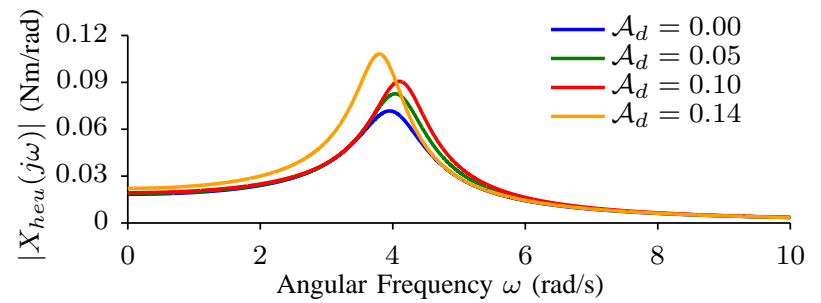

(a)

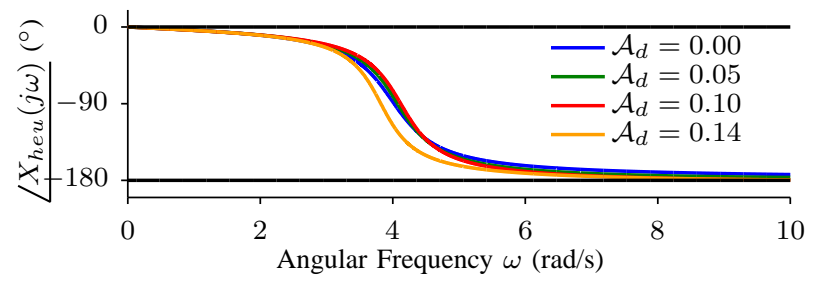

(b)

Fig. 9. The coupled human-exoskeleton system with optimal control parameters that achieve coupled stability and passivity: (a) Integral admittance magnitude $\left|X_{h e u}(j \omega)\right|,(b)$ Integral admittance phase $/ X_{h e u}(j \omega)$.

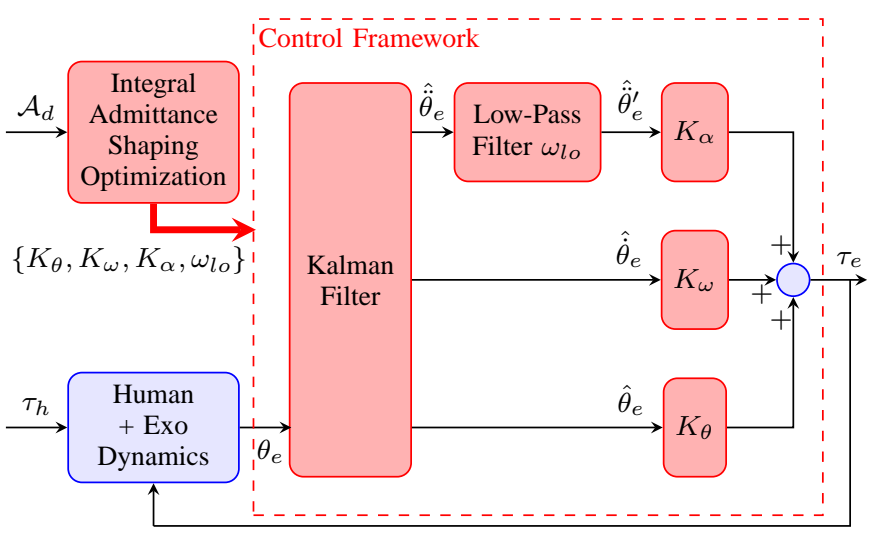

Fig. 10. Framework used for the integral admittance shaping control.

parameters from integral admittance shaping described in Sec. III. A Kalman filter based on [22] is used to filter the exoskeleton joint angle $\hat{\theta}_{e}$ and estimate angular velocity $\hat{\theta}_{e}$ and angular acceleration $\hat{\ddot{\theta}}_{e}$ needed to implement the control law in (27).

We conducted experiments on a male human subject $165 \mathrm{~cm}$ tall weighing $65 \mathrm{~kg}$. We instructed the subject to walk at his normal pace in a straight line for about $14 \mathrm{~m}$ for each of the following four cases: passive device with no assist, zero desired assist, i.e., $\mathcal{A}_{d}=0\left(\left|X_{\text {heu }}(j \omega)\right|=\left|X_{h}(j \omega)\right|\right)$, and two non-zero desired assists $\left(\mathcal{A}_{d}=0.02,0.05\right)$. Figure $11(a)$ presents the hip joint phase plots $\left(\hat{\theta}_{e}\right.$ vs $\left.\hat{\dot{\theta}}_{e}\right)$ for the four cases, and their corresponding exoskeleton torque trajectories are shown in Fig. 11(b).

It is interesting to see from Fig. 11(a) that the phase plot corresponding to $\mathcal{A}_{d}=0$ is bigger than that with just the passive exoskeleton. This confirms that the passive exoskeleton indeed weighs the human leg down and shrinks its phase plot, whereas with $\mathcal{A}_{d}=0$, the active exoskeleton is able to recover the human's original joint dynamics. Moreover, it can be clearly seen from Fig. 11(a) that the

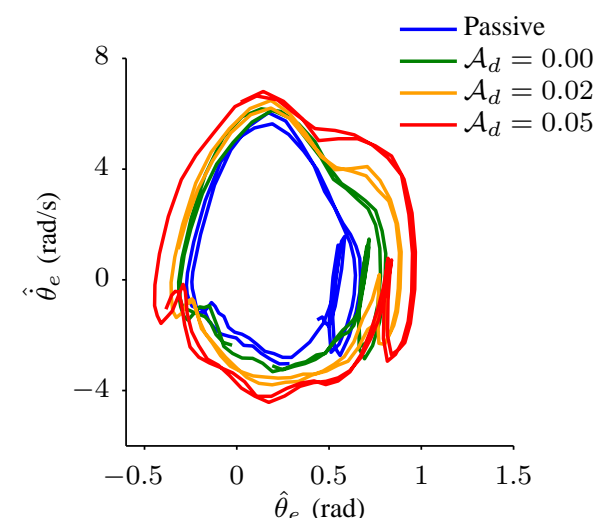

(a)

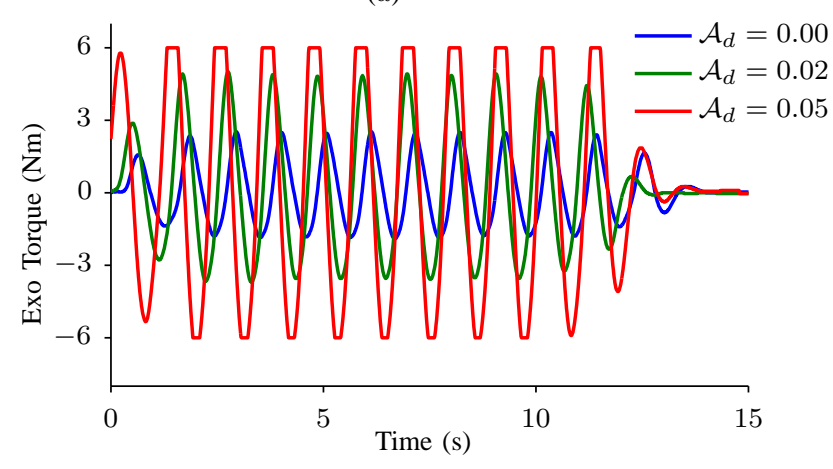

(b)

Fig. 11. Experimental results for different desired assistance ratios for a male human subject $165 \mathrm{~cm}$ tall weighing $65 \mathrm{~kg}$ : (a) hip joint phase plots, and $(b)$ exoskeleton joint torque trajectories.

phase plot grows with increasing assistance from $\mathcal{A}_{d}=0$ to $\mathcal{A}_{d}=0.05$. Therefore, as expected, the integral admittance shaping control framework amplifies the motion of the hip joint, and the level of amplification increases with increasing assistance. Figure 11(b) shows that the exoskeleton torque trajectory corresponding to $\mathcal{A}_{d}=0.05$ saturates at $6 \mathrm{Nm}$, and hence controllers achieving desired assistance ratios $\mathcal{A}_{d}>0.05$ were not tested on the device.

\section{Conclusions AND Discussions}

We presented conceptual and quantitative definitions for assistance and resistance based on the frequency response of the integral admittance of 1-DOF exoskeleton devices. An exoskeleton is considered assistive if it increases the admittance of the coupled human-exoskeleton system above that of the unassisted human resulting in motion amplification and torque reduction. We presented Integral Admittance Shaping, a unified control design framework that shapes the frequency response magnitude profile of the integral admittance of the coupled human-exoskeleton joint such that the desired assistance is achieved. Our framework also ensures that the coupled system is stable and passive, which ensures stable interaction with passive environments. We also presented experimental results on a modified version of Honda's Stride Management Assist device that demonstrated successful motion amplification of the assisted hip joint during walking. 
We now briefly discuss a few important facts that are useful in designing exoskeleton controllers using our framework. For a given desired assistance ratio, an infinite number of integral admittance shapes can achieve it, and choosing between these shapes is non-trivial. Therefore, the designer can use other metrics and even other constraints in (28) to make a unique selection from the set of infinite solutions. Integral admittance shaping is a general framework which is not limited by the definitions of assistance presented in this paper. For example, it can be used to design resistive exoskeleton controllers that offer no assistance at all and resist all human motion. Integral admittance shaping can also be used to design exoskeleton controllers that increase natural frequency of the swinging leg, which results in assistance at higher frequencies and resistance at lower frequencies.

\section{FUTURE WORK}

The future work involves extending the definitions of assistance and resistance to multiple degrees of freedom. Moreover, the definitions and approach presented here can also be extended to include task-level assistance rather than joint-level assistance. It will also be interesting to experimentally study the effect of different integral admittance shapes on different performance metrics like metabolic cost, walking speed increase, etc.

\section{APPENDIX I \\ SYSTEM PARAMETERS}

Table I presents the system parameters of the coupled human-exoskeleton system used in the analysis and experimental results presented in the paper. The human limb data were obtained from biomechanics literature [23], [24], [25], while the exoskeleton parameters were obtained from system identification experiments.

TABLE I

COUPLED Human-ExosKeleton System PARAMETERS

\begin{tabular}{|c|c|c|}
\hline Parameters & Symbol & Value \\
\hline Human Leg Length & $l_{h}$ & $0.875 \mathrm{~m}$ \\
\hline $\begin{array}{c}\text { Human Leg } \\
\text { Moment of Inertia }\end{array}$ & $I_{h}$ & $3.381 \mathrm{~kg} \cdot \mathrm{m}^{2}$ \\
\hline $\begin{array}{c}\text { Human Hip Joint } \\
\text { Damping Coefficient }\end{array}$ & $b_{h}$ & $3.5 \mathrm{~N} \cdot \mathrm{m} \cdot \mathrm{s} / \mathrm{rad}$ \\
\hline $\begin{array}{c}\text { Human Hip Joint } \\
\text { Stiffness Coefficient }\end{array}$ & $k_{h}$ & $54.677 \mathrm{~N} \cdot \mathrm{m} / \mathrm{rad}$ \\
\hline $\begin{array}{c}\text { Exoskeleton Arm } \\
\text { Moment of Inertia }\end{array}$ & $I_{e}$ & $0.01178 \mathrm{~kg} \cdot \mathrm{m}^{2}$ \\
\hline $\begin{array}{c}\text { Exoskeleton Joint } \\
\text { Damping Coefficient }\end{array}$ & $b_{e}$ & $0.34512 \mathrm{~N} \cdot \mathrm{m} \cdot \mathrm{s} / \mathrm{rad}$ \\
\hline $\begin{array}{c}\text { Exoskeleton Joint } \\
\text { Stiffness Coefficient }\end{array}$ & $k_{e}$ & $0.33895 \mathrm{~N} \cdot \mathrm{m} / \mathrm{rad}$ \\
\hline $\begin{array}{c}\text { Coupling } \\
\text { Damping Coefficient }\end{array}$ & $b_{c}$ & $9.474 \mathrm{~N} \cdot \mathrm{m} \cdot \mathrm{s} / \mathrm{rad}$ \\
\hline $\begin{array}{c}\text { Coupling } \\
\text { Stiffness Coefficient }\end{array}$ & $k_{c}$ & $1905.043 \mathrm{~N} \cdot \mathrm{m} / \mathrm{rad}$ \\
\hline
\end{tabular}

\section{REFERENCES}

[1] A. M. Dollar and H. Herr, "Lower extremity exoskeletons and active orthoses: Challenges and state-of-the-art," IEEE Trans. Robotics, vol. 24 , no. 1 , pp. 144-158, 2008.

[2] D. P. Ferris, "The exoskeletons are here," Journal of Neuroengineering and Rehabilitation, vol. 6, no. 17, pp. 1-3, 2009.

[3] G. S. Sawicki and D. P. Ferris, "Mechanics and energetics of level walking with powered ankle exoskeletons," J. Exp. Biol., vol. 211, no. Pt. 9, pp. 1402-1413, 2008.

[4] P. Malcolm, W. Derave, S. Galle, and D. D. Clercq, "A simple exoskeleton that assists plantarflexion can reduce the metabolic cost of human walking," PLoS One, vol. 8, no. 2, p. e56137, 2013.

[5] L. M. Mooney, E. J. Rouse, and H. M. Herr, "Autonomous exoskeleton reduces metabolic cost of human walking during load carriage," Journal of Neuroengineering and Rehabilitation, vol. 11, no. 80, 2014.

[6] H. Kawamoto, S. Lee, S. Kanbe, and Y. Sankai, "Power assist method for hal-3 using emg-based feedback controller," in Proc. IEEE Int. Conf. Syst., Man, Cybern., 2003, pp. 1648-1653.

[7] K. E. Gordon, C. R. Kinnaird, and D. P. Ferris, "Locomotor adaptation to a soleus emg-controlled antagonistic exoskeleton," J. Neurophysiol., vol. 109 , no. 7, pp. 1804-1814, 2013.

[8] H. Kazerooni and R. Steger, "Berkeley lower extremity exoskeleton," ASME J. Dyn. Syst., Meas., Control, vol. 128, pp. 14-25, 2006.

[9] C. J. Walsh, K. Pasch, and H. Herr, "An autonomous, underactuated exoskeleton for load-carrying augmentation," in Proc. IEEE/RSJ Int. Conf. Intelligent Robots and Systems (IROS), 2006, pp. 4110-1415.

[10] J. A. Norris, K. P. Granata, M. R. Mitros, E. M. Byrne, and A. P. Marsh, "Effect of augmented plantarflexion power on preferred walking speed and economy in young and older adults," Gait \& Posture, vol. 25 , no. 4 , pp. 620-627, 2007.

[11] G. S. Sawicki and D. P. Ferris, "Powered ankle exoskeletons reveal the metabolic cost of plantar flexor mechanical work during walking with longer steps at constant step frequency," J. Exp. Biol., vol. 212, no. 1, pp. 21-31, 2009.

[12] S. Lee and Y. Sankai, "The natural frequency-based power assist control for lower body with hal-3," in Proc. IEEE Int. Conf. Sys. Man Cyber. (ICSMC), 2003, pp. 1642-1647.

[13] G. A.-Ollinger, J. E. Colgate, M. A. Peshkin, and A. Goswami, "Design of an active one-degree-of-freedom lower-limb exoskeleton with inertia compensation," Int. J. Robotics Research, vol. 30, no. 4, pp. 486-499, 2011.

[14] Honda, "Honda's stride management assist device," http://corporate.honda.com/innovation/walk-assist/, 2008.

[15] N. Hogan and S. O. Buerger, Impedance and Interaction Control, Robotics and Automation Handbook. CRC Press, LLC., 2005, ch. 19.

[16] J. E. Colgate, "The control of dynamically interacting systems," Ph.D. dissertation, Massachusetts Institute of Technology, Cambridge, MA, 1988.

[17] J. E. Colgate and N. Hogan, "An analysis of contact instability in terms of passive physical equivalents," in Proc. IEEE Int. Conf. Robotics and Automation (ICRA), 1989, pp. 404-409.

[18] G. A.-Ollinger, J. E. Colgate, M. A. Peshkin, and A. Goswami, "Active-impedance control of a lower-limb assistive exoskeleton," in Proc. IEEE Int. Conf. Rehabil. Robot. (ICORR), 2007, pp. 188-195.

[19] _ - "A 1-DOF assistive exoskeleton with virtual negative damping: Effects on the kinematic response of the lower limbs," in Proc. IEEE Int. Conf. Intell. Robot. Sys. (IROS), 2007, pp. 1938-1944.

[20] U. Nagarajan, G. A.-Ollinger, and A. Goswami, "Integral admittance shaping: A unified framework for active exoskeleton control design," Int. J. Robotics Research, (In preparation).

[21] J. Nelder and R. Mead, "A simplex method for function minimization," The Computer Journal, vol. 7, pp. 308-313, 1964.

[22] P. Canet, "Kalman filter estimation of angular velocity and acceleration: On-line implementation," McGill University, Montréal, Canada, Tech. Rep. TR-CIM-94-15, Nov. 1994.

[23] D. A. Winter, Biomechanics and Motor Control of Human Movement ( $4^{\text {th }}$ Edition). Wiley, 2009, p. 86.

[24] K. C. Hayes and H. Hatze, "Passive visco-elastic properties of the structures spanning the human elbow joint," European Journal Applied Physiology, vol. 37, pp. 265-274, 1977.

[25] J. Doke, J. M. Donelan, and A. D. Kuo, "Mechanics and energetics of swinging the human leg," Journal of Experimental Biology, vol. 208, pp. 439-445, 2005. 